\title{
The Intraoperative Corneal Pachymetry Changes during Accelerated Corneal Cross-linking in Progressive Keratoconus Patients with Thin Corneas
}

\author{
Serap Yurttaşer Ocak ${ }^{1}$, Mehmet Serhat Mangan², Mustafa Nuri Elçioğlu ${ }^{1}$ \\ ${ }^{1}$ Department of Ophthalmology, Okmeydanı Prof. Dr. Cemil Tascioglu Education and Research Hospital, University of Health \\ Sciences, Istanbul, Turkey \\ ${ }^{2}$ Department of Ophthalmology, Haydarpasa Numune Education and Research Hospital, Sadik Eratik Eye Institute, University of \\ Health Sciences, Istanbul, Turkey
}

\begin{abstract}
Purpose: To report the intraoperative corneal pachymetry changes during accelerated corneal cross-linking (A-CXL) in progressive keratoconus patients with thin corneas.

Methods: Thirty-six eyes (mean age, $22.26 \pm 4.02$ years) with progressive keratoconic thin corneas ( $<400 \mu$ m without epithelium) who underwent A-CXL with ultraviolet (UV)-A (UVA) $\left(9 \mathrm{~mW} / \mathrm{cm}^{2}\right)$ using isotonic riboflavin5-phosphate $0.1 \%$ with $1.1 \%$ hydroxypropyl methycellulose (RF-HPMC, MedioCROSS M) were included in this retrospective study. Intraoperative corneal pachymetric changes were noted before the procedure, after removal of epithelium, after RF-HPMC instillation, before and after UV irradiation. The mean of corneal pachymetric values were compared statistically.

Results: The mean corneal pachymetry reduced from $415.72 \pm 29.66$ to $369.50 \pm 23.45 \mu \mathrm{m}$ after removal of the epithelium $(p<0.05)$. After the application of RF-HPMC solution the mean thinnest corneal pachymetry (TCP) increased to $412.89 \pm$ $26.94 \mu \mathrm{m}$. Statistically significant increase was observed in TCP after saturation with RF-HPMC $(p=0.001)$. The mean corneal pachymetry before and after UVA irradiation was $419.86 \pm 10.41$ and $417.47 \pm 8.25 \mu \mathrm{m}$, respectively $(p>0.05)$.

Conclusions: Isotonic RF-HPMC lead to a significant increase in intraoperative mean TCP. RF-HPMC seems to be a favorable riboflavin option in keratoconus patients with thin corneas.
\end{abstract}

Key Words: Cornea, Cross linking, Keratoconus, Riboflavin

Keratoconus is a degenerative disease that leads to steep and irregular astigmatism due to progressive corneal stromal thinning with onset at puberty in most cases [1,2]. Cor-

Received: July 16, 2021 Final revision: August 1, 2021

Accepted: August 13, 2021

Corresponding Author: Serap Yurttaşer Ocak, MD. Department of Ophthalmology, Okmeydanı Prof. Dr. Cemil Tascioglu Education and Research Hospital, Kaptan Paşa, SSK Okmeydanı Hst. No:25, Istanbul 34384, Turkey. Tel: 90-505-247-7215, Fax: 90-212-221-7800, E-mail: drserapocak@gmail.com neal collagen cross-linking (CXL) is the only procedure to halt the progression of keratoconus [3]. In conventional CXL (C-CXL) procedure, after epithelium removal the cornea is saturated with iso-osmolar riboflavin solution with dextran (RF-DX) every 3 minutes for a total of 30 minutes, followed by $3 \mathrm{~mW} / \mathrm{cm}^{2}$ ultraviolet (UV)-A (UVA) application for 30 minutes [2,3]. The photo-oxidative reaction formed with combination of RF-DX and UVA creates cross-links between the collagen lamellae. These bonds increase corneal resistance $[3,4]$. In time, the efficacy and 
safety profile of the accelerated UVA irradiation protocol (A-CXL) has also been supported by clinical evidence [5-7].

The studies have shown that C-CXL application should not be performed in corneas with a thickness of $<400 \mu \mathrm{m}$ (without epithelium); otherwise, the safety of deep eye structures, including the endothelium, is at risk [8]. For corneas $<400 \mu \mathrm{m}$, various alternative methods have been published [9]. In one of the alternative treatment protocol, it is aimed to swell the corneal thickness to above $400 \mu \mathrm{m}$ before UV irradiation by using hypo-osmolar riboflavin (HORF) with RF-DX [10-12]. However, it has been shown that iso-osmolar RF-DX has a thinning effect on the cornea which is undesirable particularly for thin corneas [13-16]. Hence, although initial studies of CXL were performed with a riboflavin solution containing dextran, recent protocols for CXL have indicated the use of a riboflavin solution containing iso-osmolar hydroxypropyl methylcellulose (RF-HPMC) [17,18]. There are several studies showing that RF-HPMC has an increasing effect on corneal thickness $[15,16,18,19]$. These studies were not conducted thin corneas. In this study the changes of intraoperative thinnest corneal pachymetry (TCP) were evaluated during A-CXL protocol in thin keratoconic corneas.

\section{Materials and Methods}

The data of patients who underwent A-CXL between January 2016 to March 2018 were examined. The patients who had TCP of $<400 \mu \mathrm{m}$ after mechanical debridement of epithelium during A-CXL were included in this retrospective study. The study was approved by the ethics committee of the Prof. Dr. Cemil Tascioglu Education and Research Hospital under the principles of the Declaration of Helsinki (approval number: 200). Informed written consent was obtained from each patient before the procedure. The exclusion criteria were determined as follows: (1) corneal thickness of $>400 \mu \mathrm{m}$ (without epithelium), (2) age $<18$ years or $>40$ years, (3) existence of corneal scars or opacities, (4) previous corneal or anterior segment surgery, and (5) existence of any ocular disease except keratoconus.

In our clinic, pachymetry guided CXL is carried out on those who had thinnest corneal thickness $\leq 450 \mu \mathrm{m}$ (determined by Sirius corneal tomography). In pachymetry guided CXL; first a corneal marker was used to mark the thinnest corneal area, in guidance of the Scheimpflug tomography corneal pachymetry map (Sirius; CSO Inc., Firenze, Italy). In pachymetry guided CXL, the all TCP measurements were applied by contact ultrasound pachymetry (Pocket II; Quantel Medical, Cedex, France). The measurements were taken before the procedure, after removal of epithelium, after RF-HPMC instillation, before and after UV irradiation. Five measurements had done which were required for the device in order to calculate the average pachymetry value. The intraoperative corneal pachymetric changes were evaluated statistically.

\section{Surgical technique}

Corneal cross-linking was performed under topical anesthesia with $0.5 \%$ proparacaine hydrochloride (Alcaine; Alcon, Hunenberg, Switzerland) under sterile conditions. After a region of the corneal epithelium with a diameter of $8 \mathrm{~mm}$ was removed, cornea was saturated with isotonic RF-HPMC solution (riboflavin5-phosphate $0.1 \%$ with $1.1 \%$ hydroxypropyl methycellulose; MedioCROSS M, MedioHaus Medizinprodukte, Kiel, Germany) at every 2 minutes for a total of 20 minutes. If TCP reached to a value of $\geq 400$ $\mu \mathrm{m}$ at this stage, irradiation was started. If TCP was still $<400 \mu \mathrm{m}$, HO-RF (hypo-osmolar riboflavin $310 \mathrm{mosm} / \mathrm{L}$, $0.9 \%$; MedioCROSS H, Medio-Haus Medizinprodukte) was applied at every 20 seconds for 5 minutes for corneal swelling until the TCP value reached $\geq 400 \mu \mathrm{m}$. The cornea was then exposed to $370 \mathrm{~nm}$ continuous UVA light with the A-CXL system (Vario 365; Peschke Meditrade, Hunenberg, Switzerland) for 10 minutes at an irradiance level of $9 \mathrm{~mW} /$ $\mathrm{cm}^{2}$. The RF-HPMC solution was applied at every 2 minutes during irradiation. The cornea was irrigated with cold saline and a bandage contact lens was inserted.

\section{Statistical analysis}

The NCSS 2007 (NCSS, Kaysville, UT, USA) was used for statistical analysis. Visual acuity was expressed in logMAR notation. Descriptive statistical methods (mean, standard deviation, median, frequency, percentage, minimum, and maximum) were used when evaluating the study data. The suitability of quantitative data for normal distribution was tested with the Shapiro-Wilk test and graphical examinations. In the group comparisons of quantitative variables with normal distribution, repeated measures variance analysis was used. The Friedman test was used for intragroup 
Table 1. Intraoperative thinnest corneal pachymetry measurements (micron)

\begin{tabular}{lccc}
\hline Variable & Median (Q1-Q3) & Mean \pm standard deviation & $p$-value \\
\hline Preoperative & $419(394-434)$ & $415.72 \pm 29.66$ & - \\
Epi-off & $373(354-388)$ & $369.50 \pm 23.45$ & - \\
After RF-HPMC & $410(389-421)$ & $412.89 \pm 26.94$ & - \\
Pre-UVA & $417(410-421)$ & $419.86 \pm 10.41$ & - \\
Post-UVA & $416(412-423)$ & $417.47 \pm 8.25$ & - \\
Difference & - & & $0.001^{\dagger}$ \\
Epi-off and after RF-HPMC & - & $33.39 \pm 11.15$ & $0.110^{*}$ \\
Pre-UVA and post-UVA & $-1.39 \pm 5.08$ & \\
\hline
\end{tabular}

Epi-off $=$ after epithelial removal; RF-HPMC $=$ riboflavin with hydroxypropyl methylcellulose; UVA = ultraviolet A.

${ }^{*}$ Wilcoxon signed ranks test; ${ }^{\dagger} p<0.01$; ${ }^{\star}$ Paired samples test.

comparisons of quantitative variables that did not show normal distribution. A dependent group $t$-test was used for intragroup comparisons of quantitative variables with normal distribution. Wilcoxon signed-rank test was used for intragroup comparison of quantitative variables that did not show normal distribution. Statistical significance was accepted as $p<0.05$.

\section{Results}

Thirty-six eyes from 36 patients (19 males, 17 females) were included in this study. The mean age was $22.26 \pm 4.02$ years (range, 18-36 years). Table 1 and Fig. 1 show the intraoperative changes in TCP measurements. The mean TCP reduced from $415.72 \pm 29.66$ to $369.50 \pm 23.45 \mu \mathrm{m}$ after removal of epithelium. The mean TCP was $412.89 \pm$ $26.94 \mu \mathrm{m}$ after RF-HPMC application for 20 minutes. Statistically significant increase was observed in TCP after saturation with RF-HPMC for 20 minutes (mean difference, $33.39 \pm 11.15 \mu \mathrm{m} ; p=0.001$ ). TCP was increased above $400 \mu \mathrm{m}$ with RF-HPMC in 32 of 36 eyes (88.9\%). The mean TCP before and after UVA irradiation were $419.86 \pm 10.41$ and $417.47 \pm 8.25 \mu \mathrm{m}$, respectively. There was no statistically significant difference between mean TCP values before and after UVA irradiation $(p>0.05)$. Swelling with HO-RF had to be performed in four patients (11.1\%). The mean TCP values for these four cases before the procedure, after removal of epithelium, after RF-HPMC instillation, before and after UV irradiation were $397.03 \pm 0.2,357.01 \pm 0.1,390.2 \pm 1.1,412.1 \pm 1.3,413.4 \pm 1.2$ $\mu \mathrm{m}$, respectively.

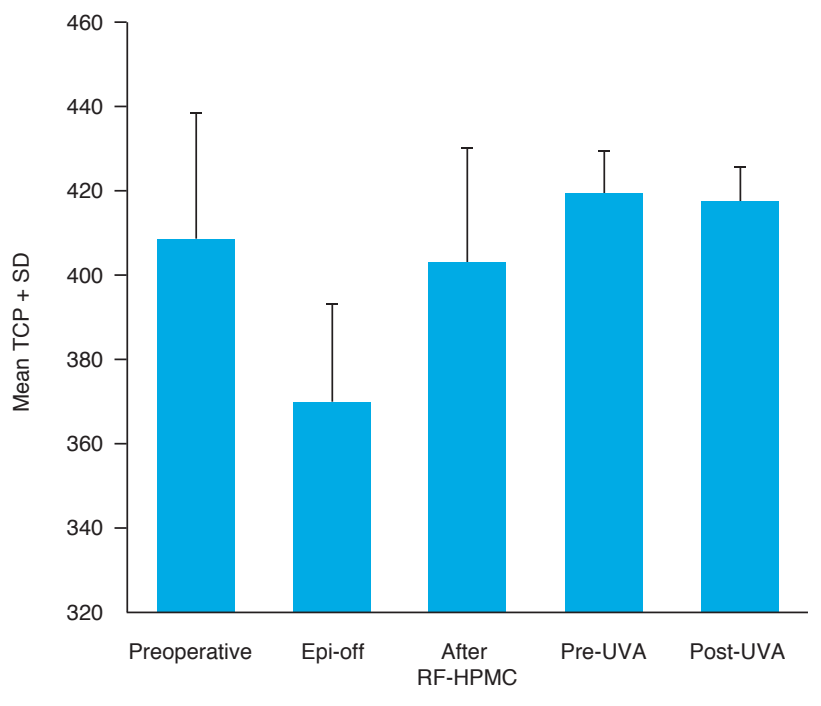

Fig. 1. Intraoperative thinnest corneal pachymetry (TCP) measurements. $\mathrm{SD}=$ standard deviation; Epi-off $=$ after epithelial removal; RF-HPMC = riboflavin with hydroxypropyl methylcellulose; UVA = ultraviolet $\mathrm{A}$.

\section{Discussion}

During the initially defined CXL technique, the corneal thickness without epithelium should be at least $400 \mu \mathrm{m}$ for the safety of endothelium and deeper ocular tissues [20]. Kymionis et al. [21] have reported significant endothelial loss after CXL applications in keratoconus cases with thin corneas. However, in many keratoconus patients, the corneal thickness is $<400 \mu \mathrm{m}$ at the time of diagnosis [22]. Various alternative methods have been published for $<400 \mu \mathrm{m}$ corneas [9]. No standard treatment protocol has been delineated. The aim of this study was to evaluate the 
changes of intraoperative TCP during A-CXL protocol in thin keratoconic corneas.

In conventional cross-linking procedure, iso-osmolar RF-DX is used in two stages. The first is the corneal saturation stage as a photosensitizer to induce the crosslinking and the second is the UVA irradiation stage to protect the underlying tissues from the damage caused by UVA light. In a method used in thin corneas, HO-RF is used in addition to RF-DX to swell the cornea before UVA irradiation stage [10-12]. However, various studies have shown the corneal thinning effect of RF-DX due to its high oncotic pressure [13-16]. This effect is an unfavorable situation particularly for thin corneas. There are studies which compared corneal thickness changes using RF-HPMC or RFDX $[15,16]$. These studies reported that although both riboflavin solutions are iso-osmolar, RF-HPMC maintained a steady increase in the corneal thickness as opposed to RFDX. The rate of increase in corneal thickness has been reported as approximately $10 \%$ in most publications (range, 5\%-26\%) $[15,16,18,19]$. It has been shown that RF-HPMC creates a thicker preocular film due to its high viscosity, compared to RF-DX [23]. This thicker film might influence the increase in corneal thickness by preventing evaporation from the cornea. However, Rechichi et al. [24] demonstrated a reduction in TCP after saturation by RF-HPMC solution. But this reducation was not statistically significant. However this result may be due to shorter RFHMPM saturation phase in their study (10 minutes). There is only one study conducted with 14 cases reported no change in corneal thickness by RF-HPMC [25]. In our study similar to most studies in the literature, we detected a mean increase of $33.39 \pm 11.15 \mu \mathrm{m}(9.03 \%)$ in TCP value after iso-osmolar HPMC-RF instillation. The TCP value increased to a safe value of $400 \mu \mathrm{m}$ in $88.9 \%$ of the eyes (32 / 36) without necessitating swelling of the cornea with HO-RF. Therefore, the use of iso-osmolar RF-HPMC for the saturation stage in thin corneas led to a reduction in the number of corneas requiring swelling with HO-RF before irradiation. We think that this is an advantage especially for thin corneas and gives us an opportunity to apply cross-linking with a single riboflavin solution.

At the end of the saturation phase, for the eyes that we could not reach to $400 \mu \mathrm{m}$ with RF-HPMC (4 / 36), we used HO-RF to provide corneal swelling until we reached to a minimum of $400 \mu \mathrm{m}$ thickness. Some studies demonstrated that artificial swelling by HO-RF was transient, since they found a reduction in TCP during the irradiation phase $[11,12,15]$. In these studies, a reduction in TCP may be the result of RF-DX used in the UV stage, due to its high oncotic pressure and thinning effect. We did not find statistically significant difference in the mean TCP values between pre- and post-UVA irradiation. The use of RF-HPMC instead of RF-DX during UVA irradiation may have prevented the thinning of TCP and it may have continued to maintain the corneal thickness throughout the procedure in thin corneas. However, there are studies which used RF-HPMC in irradiation stage and they reported an increase in the mean TCP values after UVA irradiation, compared to pre-irradiation $[15,18]$. But these studies did not include thin corneas and the measurement had been taken as central corneal thickness (CCT), not TCP.

Hagem et al. [18] compared the intraoperative CCT changes between A-CXL (10 minutes) and CXL (30 minutes) using RF-HPMC in non-thin corneas and they showed increasing in mean CCT after UV irradiation for both of the procedure. There was no statistically significant difference in mean CCT values between the two groups. The reason why they did not detect a decrease in corneal pachymetry may be prevention of dehydration by RF-HPMC due to its prolonged break-up time. There is no similar comparative study with thin corneas. Our findings also showed no significant difference in TCP after accelerated UV irradiation in thin corneas.

A limitation of this study was to locate the thinnest point on the cornea with assumption under guidance of corneal tomography, using corneal thickness map. This may limit an objective analysis of the thinnest point of the cornea.

In conclusion, using iso-osmolar RF-HPMC causes an increase in the thinnest corneal thickness after the saturation phase, hence this is offering an advantage to improve the safety of the procedure in thin corneas. RF-HPMC may also reduce the need of HO-RF in thin corneas. Therefore, these results might be beneficial in broadening the spectrum of cross-linking indications in patients with thin corneas without the need to swell the cornea with HO-RF.

\section{Conflict of Interest}

No potential conflict of interest relevant to this article was reported. 


\section{References}

1. Krachmer JH, Feder RS, Belin MW. Keratoconus and related noninflammatory corneal thinning disorders. Surv Ophthalmol 1984;28:293-322.

2. Al-Yousuf N, Mavrikakis I, Mavrikakis E, Daya SM. Penetrating keratoplasty: indications over a 10 year period. $\mathrm{Br} J$ Ophthalmol 2004;88:998-1001.

3. Wollensak G, Spoerl E, Seiler T. Riboflavin/ultraviolet-a-induced collagen crosslinking for the treatment of keratoconus. Am J Ophthalmol 2003;135:620-7.

4. Spoerl E, Wollensak G, Seiler T. Increased resistance of crosslinked cornea against enzymatic digestion. Curr Eye Res 2004;29:35-40.

5. Kymionis GD, Grentzelos MA, Kankariya VP, et al. Safety of high-intensity corneal collagen crosslinking. J Cataract Refract Surg 2014;40:1337-40.

6. Schumacher S, Oeftiger L, Mrochen M. Equivalence of biomechanical changes induced by rapid and standard corneal cross-linking, using riboflavin and ultraviolet radiation. Invest Ophthalmol Vis Sci 2011;52:9048-52.

7. Gatzioufas Z, Richoz O, Brugnoli E, Hafezi F. Safety profile of high-fluence corneal collagen cross-linking for progressive keratoconus: preliminary results from a prospective cohort study. J Refract Surg 2013;29:846-8.

8. Spoerl E, Mrochen M, Sliney D, et al. Safety of UVA-riboflavin cross-linking of the cornea. Cornea 2007;26:385-9.

9. Deshmukh R, Hafezi F, Kymionis GD, et al. Current concepts in crosslinking thin corneas. Indian J Ophthalmol 2019;67:8-15.

10. Hafezi F, Mrochen M, Iseli HP, Seiler T. Collagen crosslinking with ultraviolet-A and hypoosmolar riboflavin solution in thin corneas. J Cataract Refract Surg 2009;35:621-4.

11. Kaya V, Utine CA, Yilmaz OF. Intraoperative corneal thickness measurements during corneal collagen cross-linking with hypoosmolar riboflavin solution in thin corneas. Cornеa 2012;31:486-90.

12. Schmidinger G, Pachala M, Prager F. Pachymetry changes during corneal crosslinking: effect of closed eyelids and hypotonic riboflavin solution. J Cataract Refract Surg 2013;39:1179-83.

13. Mazzotta C, Caragiuli S. Intraoperative corneal thickness measurement by optical coherence tomography in keratoconic patients undergoing corneal collagen cross-linking. Am J Ophthalmol 2014;157:1156-62.

14. Kymionis GD, Kounis GA, Portaliou DM, et al. Intraopera- tive pachymetric measurements during corneal collagen cross-linking with riboflavin and ultraviolet A irradiation. Ophthalmology 2009;116:2336-9.

15. Zaheer N, Khan WA, Khan S, Khan MA. Comparison of changes in central corneal thickness during corneal collagen cross-linking, using 1sotonic riboflavin solutions with and without dextran, in the treatment of progressive keratoconus. Cornea 2018;37:340-6.

16. Oltulu R, Satirtav G, Donbaloglu M, et al. Intraoperative corneal thickness monitoring during corneal collagen cross-linking with isotonic riboflavin solution with and without dextran. Cornea 2014;33:1164-7.

17. Rapuano PB, Mathews PM, Florakis GJ, et al. Corneal collagen crosslinking in patients treated with dextran versus isotonic hydroxypropyl methylcellulose (HPMC) riboflavin solution: a retrospective analysis. Eye Vis (Lond) 2018;5:23.

18. Hagem AM, Thorsrud A, Sandvik GF, et al. Collagen crosslinking with conventional and accelerated ultraviolet-A irradiation using riboflavin with hydroxypropyl methylcellulose. J Cataract Refract Surg 2017;43:511-7.

19. Akkaya Turhan S, Aydin FO, Toker E. Effect of riboflavin solution with hydroxypropyl methylcellulose and eyelid speculum on pachymetry changes during accelerated collagen crosslinking. Cornea 2019;38:864-7.

20. Koc M, Uzel MM, Koban Y, et al. Comparison of results of accelerated corneal cross-linking with hypo-osmolar riboflavin solution performed on corneas thicker and thinner than $400 \mu \mathrm{m}$. Cornea 2016;35:151-6.

21. Kymionis GD, Portaliou DM, Diakonis VF, et al. Corneal collagen cross-linking with riboflavin and ultraviolet-A irradiation in patients with thin corneas. Am J Ophthalmol 2012;153:24-8.

22. Koc M, Uzel MM, Koban Y, et al. Accelerated corneal cross-linking with a hypoosmolar riboflavin solution in keratoconic thin corneas: short-term results. Cornea 2016;35:350-4.

23. Wollensak G, Aurich H, Wirbelauer C, Sel S. Significance of the riboflavin film in corneal collagen crosslinking. $J$ Cataract Refract Surg 2010;36:114-20.

24. Rechichi M, Mazzotta C, Daya S, et al. Intraoperative OCT pachymetry in patients undergoing dextran-free riboflavin UVA accelerated corneal collagen crosslinking. Curr Eye Res 2016;41:1310-5.

25. Jain V, Gazali Z, Bidayi R. Isotonic riboflavin and HPMC with accelerated cross-linking protocol. Cornea 2014;33:910-3. 